\title{
O olhar das pessoas com deficiência visual sobre o acesso à informação nos cuidados de saúde primários ${ }^{1}$
}

\section{Visually impaired people's outlook on primary health care information}

\author{
RITA PEREIRA \\ Mestre em Comunicação Acessível \\ Coordenadora dos serviços de reabilitação e ação social, ACAPO, Portugal \\ rita.apc@gmail.com
}

CARLA FREIRE

Doutorada em Engenharia Multimédia

Professora adjunta na ESECS, Politécnico de Leiria, Portugal

Investigadora integrada no CI\&Dei e colaboradora no CICS.NOVA.IPLeiria-iACT, Portugal

carla.freire@ipleiria.pt

\section{RESUMO}

Considerando os direitos da pessoa com deficiência visual e a necessidade do ambiente se adaptar à diversidade de públicos, pretende-se compreender em que medida os centros de saúde de Portugal respondem às suas necessidades de comunicação e de interação no acesso aos cuidados de saúde primários. Os dados deste estudo, exploratório-descritivo, resultaram do preenchimento de um inquérito por questionário 124 pessoas com deficiência visual. Conclui-se que os centros de saúde não respondem às necessidades da maioria dos participantes, sendo as consequências deste facto a perda de autonomia, privacidade e informações importantes, para além de serem, também, percecionadas algumas barreiras que se prendem a questões interpessoais. Os resultados obtidos reforçam a importância de sensibilizar, por um lado, as pessoas com deficiência visual para a afirmação esclarecida dos seus direitos e por outro, os profissionais e organizações sobre o impacto da deficiência visual nas dimensões interpessoais e na autogestão das informações.

Palavras-chave: Informação acessível. Comunicação acessível. Literacia em saúde. Cuidados de saúde primários. Pessoas com deficiência visual.

\begin{abstract}
Considering the rights of people with visual impairment and the need for the environment to adapt to the diversity of people, it is intended to understand the extent to which health centres of Portugal respond to communication and interaction needs of people with visual impairment when they access to primary health care. The data collected in this exploratory-descriptive study resulted from a questionnaire survey, by 124 people with visual impairment. It is possible to conclude that health centers do not meet the needs of the majority of participants, and the consequences of this fact are the loss of autonomy, privacy and important information, besides also being perceived some barriers related to interpersonal issues. The results reinforce the importance of awareness of people with visual impairment to their rights and, on the other hand, professionals and organizations about the impact of visual impairment on interpersonal dimensions and self-management of information.
\end{abstract}

Keywords: Accessible information. Accessible communication. Health literacy. Primary health care. Visually impaired people.

\footnotetext{
${ }^{1}$ Artigo submetido para avaliação em 19/05/2021 e aprovado em 16/ 06/2021.
} 
Rev. Interd. em Cult. e Soc. (RICS), São Luís, v. 7, n. 1, p. 225 - 241, jan./jun. 2021

ISSN eletrônico: 2447-6498

\section{INTRODUÇÃO}

A Convenção sobre os Direitos das Pessoas com Deficiência (CDPD) reconhece, no seu artigo $25^{\circ}$, o direito das pessoas com deficiência ao gozo do melhor estado de saúde possível, assim como o acesso ao mais alto padrão de cuidados de saúde sem discriminação, devendo os Estados Partes providenciar-lhes a mesma gama, qualidade e padrão de serviços de acordo com os prestados às restantes pessoas (Resolução da Assembleia da República n. ${ }^{\circ}$ 56/2009). Contudo, para que se verifique este direito, é necessário criar condições que permitam à pessoa com deficiência viver de modo independente e participar plenamente em todos os aspetos da vida, nomeadamente, de formas que permitam o acesso aos ambientes físicos, aos transportes, aos serviços prestados ao público e à mobilidade pessoal com a maior independência possível, à liberdade de expressão e ao acesso à informação por meio de todas as formas de comunicação da sua escolha, no que se refere a formatos e tecnologia disponíveis. Destaca a referida Resolução - como exemplo a seguir pelos Estados Partes “[...] a prestação de informação destinada ao público em geral, às pessoas com deficiência, em formatos e tecnologias acessíveis apropriados aos diferentes tipos de deficiência, de forma atempada e sem qualquer custo adicional” (MINISTÉRIO PÚBLICO DE PORTUGAL, 2009, p.10).

A literacia em saúde influencia a decisão de um indivíduo relativamente à sua saúde ou daqueles que lhe são próximos, afetando a qualidade de vida e podendo resultar num aumento de despesas médicas. (ESPANHA, ÁVILA; MENDES, 2016). Em Portugal, 5 em cada 10 indivíduos têm baixos níveis de literacia em saúde (ARRIAGA, SANTOS, SILVA, MATA, CHAVES; FREITAS, 2019), o que se traduz num número significativo de pessoas que têm dificuldade em compreender e utilizar adequadamente informações sobre a saúde. Espanha, Ávila e Mendes (2016) identificaram alguns grupos vulneráveis (e.g. pessoas com mais de 66 anos, baixos níveis de escolaridade, baixos rendimentos), cujo nível de literacia é considerado problemático ou inadequado. Apesar de o estudo destacar grupos vulneráveis, não identificou a população com deficiência, nomeadamente as pessoas com Deficiência Visual (DV), que para além de poderem ter dificuldade na compreensão da informação, podem ter, também, dificuldade no acesso quando não existem ajustes que permitam a acessibilidade à informação escrita e ao espaço físico.

Neste sentido, considerando que a "Literacia em Saúde implica o conhecimento, a motivação e as competências das pessoas para aceder, compreender, avaliar e aplicar infor- 
Rev. Interd. em Cult. e Soc. (RICS), São Luís, v. 7, n. 1, p. 225 - 241, jan./jun. 2021

ISSN eletrônico: 2447-6498

mação em saúde de forma a formar juízos e tomar decisões no quotidiano sobre cuidados de saúde" (ARRIAGA et al, 2019, p.6), é imperativo compreender se o centro de saúde (CS) responde às necessidades de comunicação das pessoas com DV nos cuidados de saúde primários. É importante analisar em que medida os suportes de informação disponibilizados por estes serviços respondem às necessidades de leitura das pessoas com DV e descrever a perceção destas pessoas no que concerne à comunicação e à interação direta com os profissionais de saúde. Conhecendo estas dimensões, com base na experiência das pessoas com DV enquanto utentes, será mais fácil providenciar os ajustes que permitam o seu acesso autónomo aos serviços de saúde, à literacia em saúde e, consequentemente, à melhoria da qualidade de vida.

\section{O ACESSO DA PESSOA COM DEFICIÊNCIA VISUAL À SAÚDE: DESAFIOS E POTENCIAIS FACILITADORES}

Para compreender as implicações que podem existir no acesso à saúde, por parte das pessoas com DV, é importante contextualizar o conceito, neste sentido, entende-se por acessibilidade um produto ou serviço que pode ser utilizado por todas as pessoas, independente da forma como o encontram. (INTERACTION DESIGN FOUNDATION, s.d.). A acessibilidade pode ser vista sob diferentes dimensões, as quais podem influenciar o acesso a algo e, consequentemente, condicionar as pessoas às suas escolhas, aos seus direitos, por toda a vida. De acordo com Sassaki (2009), existem seis dimensões que podem afetar a acessibilidade: a arquitetónica, no que se refere aos espaços físicos; a comunicacional, no que concerne à transmissão de informação; a metodológica, quanto aos métodos e técnicas adotados; a instrumental, referindo-se a instrumentos, ferramentas ou utensílios; a programática, ao nível de políticas públicas, legislação, normas, entre outras; e a atitudinal, relacionandose com preconceitos, estereótipos, estigmas e discriminações.

Apesar de o conceito de acessibilidade pressupor acesso global a produtos e serviços, de forma geral, as pessoas com DV têm dificuldade na leitura da informação médica, uma vez que esta não é facultada em formatos acessíveis, tais como em Braille ou em letras ampliadas. (SIBLEY, 2009; NZEGWU, 2005; O’DAY, KILLEEN, IEZZONI, 2004). De acordo com um estudo feito na Escócia, no qual participaram 228 indivíduos, verificou-se que apenas $10 \%$ da comunicação sobre cuidados de saúde primários foram disponibilizados em formatos de leitura preferenciais das pessoas com DV (THURSTON, THURSTON, 
Rev. Interd. em Cult. e Soc. (RICS), São Luís, v. 7, n. 1, p. 225 - 241, jan./jun. 2021

ISSN eletrônico: 2447-6498

2010), o que revela que muitas pessoas continuam sem acesso pleno à informação sobre a sua saúde.

A utilização de tecnologias, por parte das instituições médicas, pode ser uma alternativa para potencializar o acesso à informação, na medida em que muitas pessoas com DV conseguem ter acesso aos documentos por este meio, de leitores de ecrã ou de softwares de ampliação. Desta forma, terão mais facilidade em ler as informações de saúde. (SIBLEY, 2009). Contudo, é importante não esquecer que as pessoas que não utilizam tecnologia podem ficar excluídas deste acesso, pelo que urge dar respostas válidas, em formatos legíveis, a todos os cidadãos.

A literatura científica (e.g. SIBLEY, 2009; THURSTON, THURSTON, 2010) aponta consequências que a falta de informação num formato legível pode trazer para as pessoas com DV, tais como: falta de compreensão de informação sobre a própria saúde; dificuldade na tomada de decisão informada sobre a própria saúde; insegurança no ato de identificar e tomar a medicação; toma errada de medicação; perda de consultas ou exames; falta de privacidade e autonomia, na medida em que existe dependência de terceiros para a leitura de informação pessoal relativa à própria saúde.

Diversos autores consideram que para otimizar o contato entre os profissionais dos serviços de saúde e as pessoas com DV, é importante comunicar de uma forma individualizada e eficaz, antecipando dificuldades. (MEDEIROS et al 2017; CUPPLES et al, 2012; O’DAY, KILLEEN, IEZZONI, 2004). Esta comunicação deve responder às necessidades das pessoas com DV nos espaços físicos de saúde, mas também quando regressam à casa.

Refere a literatura (e.g. CUPPLES, HART, JOHNSTON, JACKSON; 2012; ASSOCIAÇÃO DOS CEGOS E AMBLÍOPES DE PORTUGAL, s.d.), e no caso concreto da DV, que três vertentes devem ser consideradas para a otimização da comunicação:

- No acesso à informação acerca das consultas e tratamentos (e.g. respeitar as preferências das pessoas com DV face ao seu suporte da informação escrita preferencial).

- Na sinalização e eliminação de barreiras físicas do ambiente (e.g. criar passagens sem obstáculos, atentar para os níveis de iluminação, usar sinalética áudio e tátil).

- Na promoção competências de comunicação e de interação (e.g. fazer contacto visual com a pessoa, independentemente da sua falta de visão, apresentando-se e falando diretamente para ela e não para o seu acompanhante). 
Rev. Interd. em Cult. e Soc. (RICS), São Luís, v. 7, n. 1, p. 225 - 241, jan./jun. 2021 ISSN eletrônico: 2447-6498

É crucial que os profissionais da saúde possibilitem a comunicação para todas as pessoas, sejam quais forem as suas características. Desta forma, devem adotar medidas universais, tais como: uso linguagem simples sem terminologias específicas e técnicas; concentração de informação estritamente necessária; repetição de informações chave; uso de ilustrações, vídeos e/ou demonstrações; concessão de documentação fácil de ler e que complemente as instruções orais; assegurar que o paciente compreendeu a informação transmitida. (PAIVA et al, 2017).

Diversificando as estratégias e os modos de comunicação, atendendo à diversidade de perfis sociais, o profissional de saúde conseguirá (para além das suas competências clínicas), de uma boa dinâmica comunicacional verbal e não-verbal fortalecer o processo de decisão crítica do utente. Diz-nos a literatura que esta adequação do profissional de saúde (e respetivos serviços) não só promove o aumento da literacia em saúde, como a eficácia e eficiência dos serviços de saúde sendo, portanto, uma ferramenta essencial para a sustentabilidade do Serviço Nacional de Saúde. (ALMEIDA et al, 2019)

Portanto, para que exista melhoria no acesso aos cuidados de saúde primários por parte das pessoas com DV, é necessário envolver todos os agentes desta área: os profissionais, as pessoas com DV e as próprias organizações. (SIBLEY,2009). Sendo fundamental que os profissionais de saúde sejam esclarecidos sobre a DV para que possam compreender as necessidades das pessoas e as possíveis consequências das informações inacessíveis. (SIBLEY, 2009; THURSTON, THURSTON, 2010).

As pessoas com DV devem ser informadas da legislação que as protege, podendo ficar mais conscientes dos seus direitos e eventualmente reivindicá-los. Devem ser instruídas a solicitar informações no formato de leitura preferencial, compreendendo que ao agirem dessa forma sensibilizarão os profissionais de saúde quanto às necessidades de leitura. (SIBLEY, 2009; THURSTON, HURSTON, 2010).

As organizações devem disseminar proativamente orientações internas sobre como responder às necessidades de informação das pessoas com DV, adequando as questões logísticas para esse fim, materiais e recursos humanos. (ASSOCIAÇÃO DOS CEGOS E AMBLÍOPES DE PORTUGAL, S.D.; SIBLEY, 2009). A acessibilidade também deve ser prioridade para qualquer entidade ao se comunicar com o público, cumprindo as recomendações da Iniciativa para a Acessibilidade da Web do World Wide Web Consortium (W3C). (AS- 
Rev. Interd. em Cult. e Soc. (RICS), São Luís, v. 7, n. 1, p. 225 - 241, jan./jun. 2021

ISSN eletrônico: 2447-6498

SOCIAÇÃO DOS CEGOS E AMBLÍOPES DE PORTUGAL, S.D.; IEZZONI, O’DAY, 2006).

\section{OPÇÕES METODOLÓGICAS}

O presente artigo apresenta um estudo exploratório-descritivo que analisa se os CS respondem às necessidades das pessoas com DV. Em particular, pretende analisar em que medida os suportes de informação disponibilizados por estes serviços respondem às necessidades de leitura das pessoas com DV e descrever a perceção destas pessoas quanto à comunicação e interação direta com os profissionais de saúde. (PEREIRA, 2020; PEREIRA, FREIRE, 2020).

Para maior profundidade e compreensão do fenómeno aqui analisado, este estudo misto pretende cruzar resultados relativos às tendências nas opções de resposta, ou seja, compreender a visão dos participantes, pessoas com DV, sobre as dificuldades que sentem no acesso aos cuidados de saúde primários e, para tal, baseia-se em conhecimento pragmático e na recolha de dados quantitativos e qualitativos. (CRESWELL, 2007).

Quanto à perceção de pessoas com DV em relação ao acesso aos cuidados de saúde primários, com o intuito de recolher dados de forma sistemática, foi utilizado um inquérito por questionário com questões de resposta fechada e questões de resposta aberta. (ALMEIDA, PINTO, 1995). Considerou-se este instrumento adequado, na medida em que possibilita o preenchimento autônomo, com potencial de chegar a várias pessoas simultaneamente, natureza impessoal, através da obtenção de respostas anónimas, e permitindo a uniformização na apresentação das questões. (FORTIN, 2009). O instrumento baseado nos estudos de O’Day, Killeen e Iezzoni (2004); Cupples, Hart, Johnston e Jackson (2012); Sibley (2009); Thurston e Thurston (2010) e Medeiros, Costa, Costa, Martins e Dantas, (2017) foi testado por professores do Politécnico de Leiria e por profissionais da área da DV.

Uma versão aperfeiçoada foi, ainda, disponibilizada a um grupo de pessoas com DV, com o intuito de avaliarem o nível de compreensão das questões e da acessibilidade. $\mathrm{O}$ questionário final subdivide-se em quatro partes: 1) caracterização dos participantes, com o intuito de conhecer os dados sociodemográficos e tipologia de DV; 2) questões relacionadas com o acesso à informação escrita e as respostas existentes nos centros de saúde a este nível. O objetivo é perceber se as preferências de leitura das pessoas com DV são correspondidas, quais os impactos da informação inacessível e de que modo a autonomia e privacidade é 
Rev. Interd. em Cult. e Soc. (RICS), São Luís, v. 7, n. 1, p. 225 - 241, jan./jun. 2021

ISSN eletrônico: 2447-6498

posta em causa; 3) questões relacionadas à especificidade da interação entre pessoas com DV e profissionais no contexto dos cuidados de saúde, interessando saber como as pessoas com DV se sentem quando são atendidas e orientadas nos centros de saúde; e 4) duas questões de resposta aberta facultativas: uma sobre iniciativas positivas dos centros de saúde que fossem importante destacar e outra sobre sugestões que possam melhorar a acessibilidade da comunicação dos centros de saúde. Este último bloco tem como objetivo identificar boas práticas e sugestões que possam vir a ser replicadas em diferentes contextos, com vista a melhorarem os serviços e o atendimento nesta área.

O presente artigo centra-se nos resultados relacionados às respostas dos CS, no que se refere à disponibilização de informação acessível e à interação das pessoas com DV e os profissionais de saúde.

Para a recolha de dados, associações de pessoas com DV foram contactadas, com o intuito destas divulgarem o inquérito por meio de email e, ao mesmo tempo, tal inquérito ser divulgado em sites de redes sociais. O inquérito foi respondido por 124 indivíduos com DV (cegueira ou baixa visão) que cumpriram com os critérios de participação no estudo: ter mais de 18 anos, ser residente em Portugal e ter ido a um CS nos últimos cinco anos.

Para tratamento dos dados quantitativos foi utilizado o software Statistical Package for Social Sciences (SPSS) versão 25 e para elaboração dos gráficos, utilizou-se o software Microsoft Excel versão 2103. A análise de conteúdo foi feita manualmente após a leitura atenta das respostas. Neste sentido, foram definidas categorias e subcategorias a posteriori (Tabela 1 e Tabela 2), que permitiram agregar vários indicadores/unidades de registo e respetivas unidades de contexto, possibilitando a organização da informação para melhor análise dos resultados.

Tabela 1 - Categorias e subcategorias da análise de conteúdo sobre os motivos pelos quais os participantes não pedem informações acessíveis

\begin{tabular}{|l|l|}
\hline Categorias & Subcategorias \\
\hline \multirow{4}{*}{ Motivo } & Necessidade \\
\cline { 2 - 2 } & Desconhece uma razão \\
\cline { 2 - 2 } & Apoio dos profissionais \\
\cline { 2 - 2 } & Recebe informação por via eletrónica \\
\cline { 2 - 2 } & Desconhecimento de direitos e/ou práticas \\
\hline
\end{tabular}


Rev. Interd. em Cult. e Soc. (RICS), São Luís, v. 7, n. 1, p. 225 - 241, jan./jun. 2021 ISSN eletrônico: 2447-6498

\begin{tabular}{|l|l|}
\hline Categorias & Subcategorias \\
\hline \multirow{4}{*}{} & Crença de que não apoiariam \\
\cline { 2 - 3 } & Carência de serviços de apoio \\
\cline { 2 - 2 } & Não foram questionados \\
\cline { 2 - 2 } & Dificuldades em ler \\
\cline { 2 - 2 } & Apoio de terceiros \\
\cline { 2 - 2 } & Receio de incomodar \\
\cline { 2 - 2 } & Proximidade do CS \\
\cline { 2 - 2 } & Frequência \\
\cline { 2 - 2 } & Uso de estratégias \\
\hline
\end{tabular}

Fonte: Elaboração própria.

Tabela 2 - Categorias e subcategorias da análise de conteúdo sobre as consequências da informação inacessível para os participantes

\begin{tabular}{|l|l|}
\hline Categorias & Subcategorias \\
\hline \multirow{4}{*}{ Em que medida se sentiu afetado } & Perda de informação \\
\cline { 2 - 2 } & Perda de autonomia \\
\cline { 2 - 2 } & Esforço acrescido \\
\cline { 2 - 2 } & Desmotivação \\
\hline
\end{tabular}

Fonte: Elaboração própria.

De forma a respeitar todas as questões éticas e princípios definidos pela Lei da proteção de dados pessoais (Regulamento, UE, 2016/679, 2016), foi feito um pedido de parecer à Comissão de Ética do Politécnico de Leiria, o qual obteve parecer positivo (N. ${ }^{\circ}$ CE/IPLEIRIA/15/2019).

\section{PRINCIPAIS RESULTADOS}

O inquérito foi respondido por 124 pessoas, das quais 56,5\% são do sexo masculino, a média de idade em torno dos 47 anos, com desvio padrão de 14,10, sendo a amplitude de idades compreendida entre os 20 e os 80 anos. No que se refere à deficiência visual, 62,1\% dos participantes dizem possuir cegueira e 37,9\% baixa visão. Quanto ao nível acadêmico, 46,8\% dos participantes têm estudos de nível superior (licenciatura, mestrado ou doutoramento), $31,5 \%$ dos inquiridos têm o $12^{\circ}$ ano e $21,8 \%$ das pessoas, o ensino básico. 
Rev. Interd. em Cult. e Soc. (RICS), São Luís, v. 7, n. 1, p. 225 - 241, jan./jun. 2021 ISSN eletrônico: 2447-6498

Os inquiridos distribuem-se por 17 distritos do território continental e pela Região Autônoma dos Açores, sendo o maior número de participantes, 25,8\% pertencentes ao distrito de Lisboa, seguindo-se Leiria com $15,3 \%$, Porto com $14,5 \%$ e Coimbra com $8,1 \%$ dos participantes.

\section{Acesso à informação escrita}

Os resultados revelam que apenas $25 \%$ dos participantes recebem informação num formato acessível, o que vai ao encontro das conclusões de Thurston e Thurston (2010), ao assegurarem que apenas $10 \%$ da comunicação dos serviços é prestada nos formatos de leitura preferenciais das pessoas com DV. Cerca de $88 \%$ dos respondentes nunca foram questionados, no seu CS, sobre o formato preferencial em que prefere receber informação de saúde. Todavia, as pessoas com DV consideram essencial receber informação sobre saúde no formato de leitura que lhes é preferencial, sendo imprescindível a disseminação da informação em formatos alternativos. (e.g. O’DAY, KILLEN, IEZZONI, 2004; NZEGWU, 2005; SIBLEY, 2009; THURSTON, THURSTON, 2010)

De acordo com os resultados apresentados no gráfico 1, o leitor de ecrã foi selecionado por $54 \%$ das pessoas como sendo o método de leitura preferencial, enquanto a utilização do Braille em papel representa a escolha de 16,1\% dos participantes e as letras ampliadas, num suporte digital, de $14,5 \%$ dos participantes.

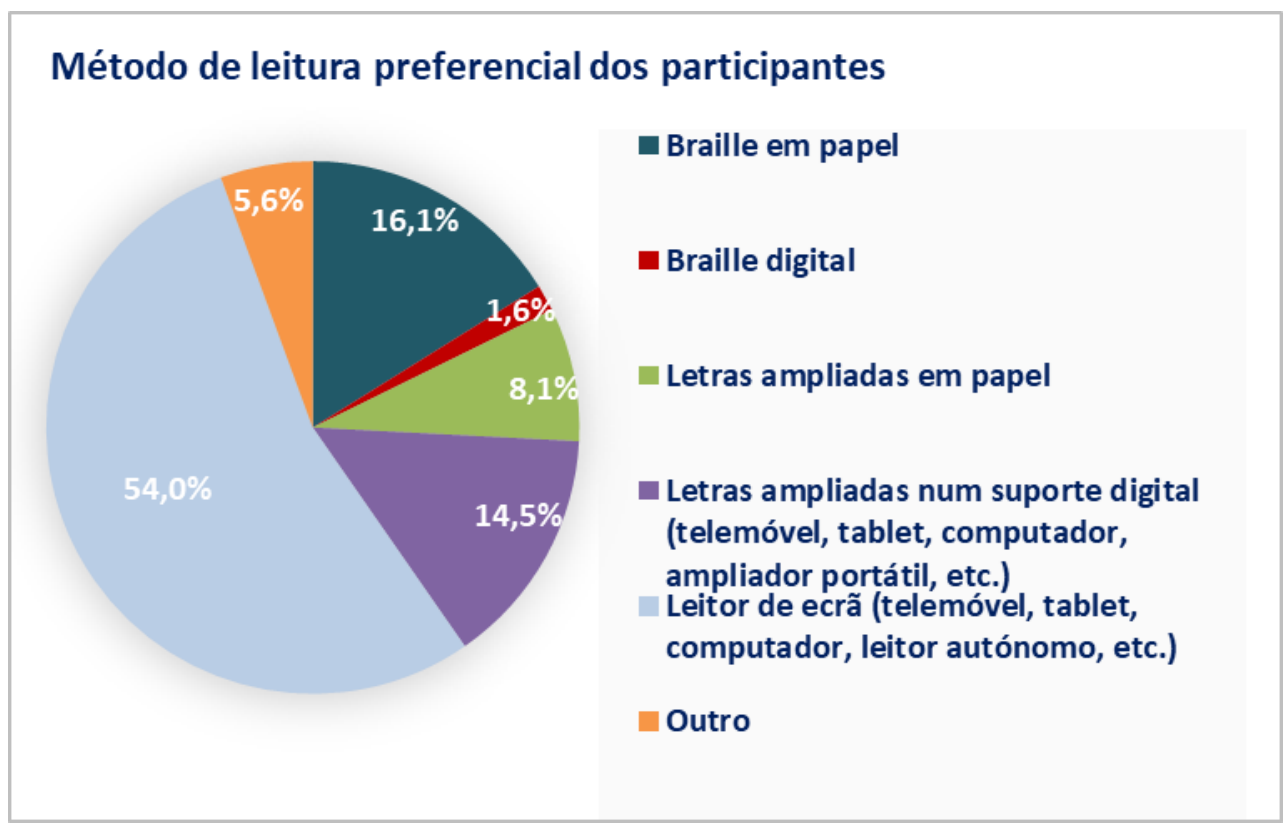

Fonte: Elaboração própria. 
Rev. Interd. em Cult. e Soc. (RICS), São Luís, v. 7, n. 1, p. 225 - 241, jan./jun. 2021 ISSN eletrônico: 2447-6498

Gráfico 1 - Informações relativas ao método de leitura preferencial dos participantes. N=124

A diferença nos pesos percentuais implica necessidade de reflexão, pois, nem todas as pessoas com DV sabem ler Braille, porque desenvolveram a DV tardiamente ou porque têm dificuldades no tato; por outro lado, a preferência pela utilização de tecnologias da informação e comunicação vai ao encontro da literatura que aponta as vantagens dos meios eletrónicos para uma comunicação mais acessível, não só em termos de leitura, mas também pela agilização da comunicação, reduzindo a distância física entre as pessoas e os profissionais. (IEZZONI, O’DAY, 2006).

No que concerne ao grupo de participantes que recebe informação acessível, 21,6\% afirmam receber a prescrição de medicamentos e $12 \%$ o agendamento de consultas, maioritariamente, em formato digital, incluindo SMS (Gráfico 2). Este fato favorece as pessoas com DV que usam formatos digitais no sentido de conseguirem ler a informação, ao contrário das que não usam as novas tecnologias (SIBLEY, 2009), tornando-se, por este motivo, um fator importante a investigar, de forma a procurar as melhores opções que permitam dar resposta a todos os cidadãos, quer utilizem, ou não, a tecnologia.

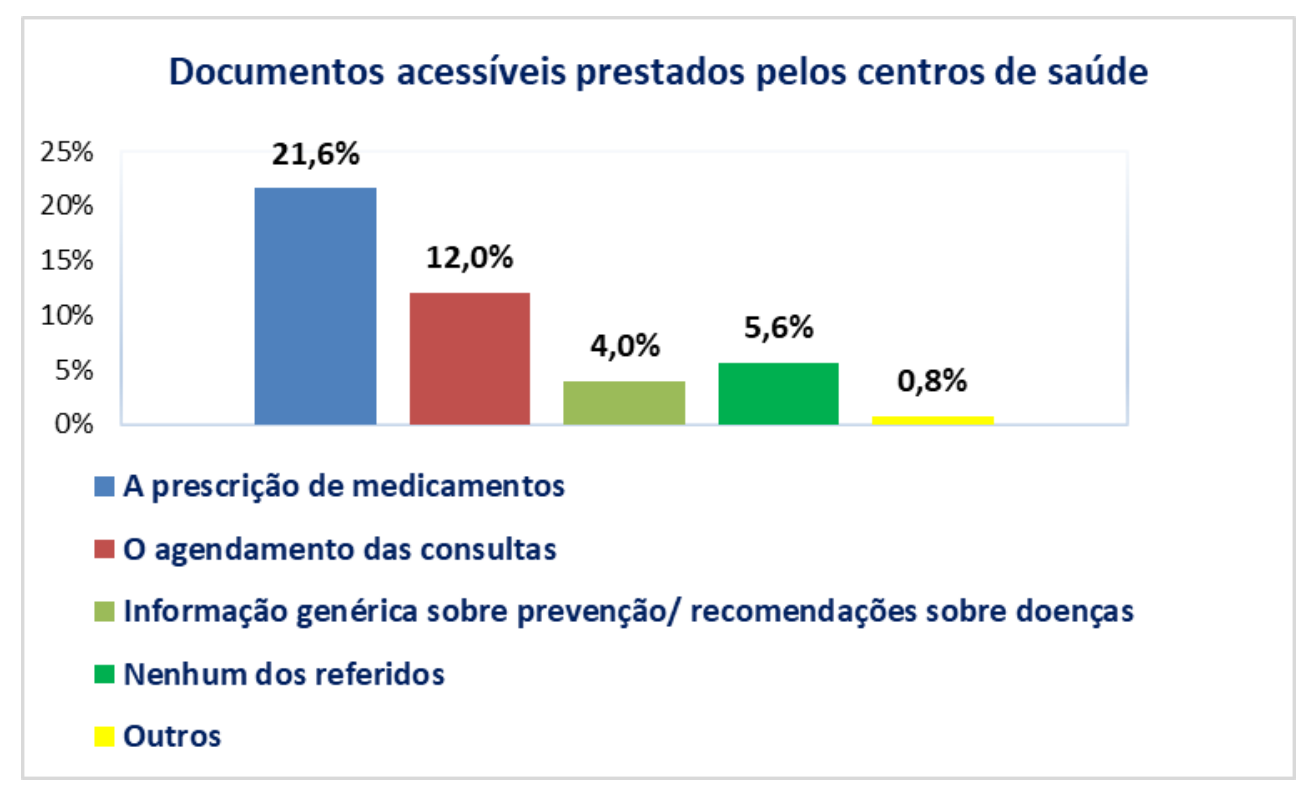

Fonte: Elaboração própria.

Gráfico 2 - Dados referentes ao tipo de documentos acessíveis prestados pelos centros de saúde.

$$
\mathrm{N}=31
$$

Por outro lado, 77\% dos inquiridos não pedem ao seu CS informação num suporte acessível por diversos motivos: não sentem necessidade, pois nunca tinham "pensado que o centro de saúde deveria de fato disponibilizar informação em formato acessível” (P132); 
Rev. Interd. em Cult. e Soc. (RICS), São Luís, v. 7, n. 1, p. 225 - 241, jan./jun. 2021 ISSN eletrônico: 2447-6498

desconhecem direitos/ legislação e/ou práticas e creem que o CS não os apoiaria a este nível; beneficiam de apoio na leitura por parte de amigos e/ou familiares; recorrem à elaboração de apontamentos, à memorização da informação prestada na consulta e/ou à utilização de produtos de apoio em casa. Devido as pessoas com DV utilizarem estratégias cognitivas e comportamentais para fazer face à inacessibilidade da informação pode justificar o porquê de não solicitarem informação em formatos acessíveis. (THURSTON; THURSTON, 2010).

Em menor escala, mas a destacar, pela necessidade de apostar na formação e na divulgação de informação sobre direitos e deveres, sublinham-se os participantes que não pedem informação acessível porque sentem ser um esforço, porque não querem incomodar os serviços e desconhecem os diferentes formatos de acesso à informação acessível. Atendendo às evidências encontradas, é mister que as instituições apresentem informações em formatos alternativos como elemento principal dos seus serviços, ou seja, como escolha dos utentes e não como requisito especial. A este respeito, Thurston e Thurston (2010) concluíram, no seu estudo, que $11 \%$ dos seus participantes não desejam solicitar informações no seu formato de leitura preferencial porque não querem ser rotuladas como diferentes. É crucial informar às pessoas com DV dos seus direitos, para que possam reivindicar com confiança o acesso à informação acessível e, desta forma, sensibilizar os profissionais de saúde para a importância das necessidades de acesso à informação por parte das pessoas com DV. (SIBLEY, 2009; THURSTON, THURSTON, 2010).

Ainda que cerca de $68 \%$ dos respondentes aleguem não se sentirem afetados pela falta de documentação acessível, $25 \%$ dos inquiridos sentem consequências desta privação. As áreas em que se sentem mais afetados referem-se à perda de informação sobre os horários da medicação, a toma/compra de medicação, o dia da consulta e informação generalizada. É também destacado como consequência, mais uma vez, o esforço acrescido: "ter de pensar com muita antecedência" (P172) e ter de confirmar sempre pelo telefone "a data e hora da marcação das consultas, por recear [esquecer-se]" (P185). Tal como nos estudos de Sibley (2009) e Thurston e Thurston (2010), existem referências à perda de autonomia pelas pessoas com DV porque dependem de terceiros e, desta forma, podem comprometer a sua capacidade de decisão, tal como defende P223 que "implica retirar parte da minha autonomia de verificação".

No que se refere à garantia de medidas de privacidade por parte dos serviços, apenas $15 \%$ dos inquiridos respondeu que essas medidas são disponibilizadas sempre ou quase 
Rev. Interd. em Cult. e Soc. (RICS), São Luís, v. 7, n. 1, p. 225 - 241, jan./jun. 2021 ISSN eletrônico: 2447-6498

sempre pelos seus Centros de Saúde, enquanto $16 \%$ das pessoas afirmam que só acontece às vezes. A partilha oral de informações, de foro pessoal, em espaços partilhados por outras pessoas pode comprometer a privacidade e confidencialidade da pessoa que se vê obrigada a responder por essa via. (IEZZONI, O’DAY, 2006). A CDPD reforça, no seu artigo 22..$^{\circ}$ o respeito, em condições de igualdade com as demais, pela proteção da confidencialidade das informações pessoais e de saúde das pessoas com deficiência.

De acordo com Sibley (2009), ter de confiar nos outros para aceder à informação sobre a saúde representa, para as pessoas com DV, uma perda de independência e privacidade. Thurston e Thurston (2010) defendem, ainda, que esta perda representa um impacto negativo em termos psicológicos. Os resultados do gráfico 3 permitem visualizar que um grupo significativo dos inquiridos $(31,5 \%)$ afirma que, às vezes, a sua autonomia é posta em causa pelo fato de outras pessoas realizarem a leitura das informações relacionadas à sua saúde, sendo que $8 \%$ respondem que isso acontece quase sempre e $11 \%$ sempre. A perda de privacidade, pelo mesmo motivo relacionado anteriormente, é igualmente considerada posta em causa, às vezes, por $26 \%$ dos participantes, sendo que um total de $6 \%$ diz acontecer "quase sempre" (8\%) e 4\% "sempre" (6\% e $8 \%)$.

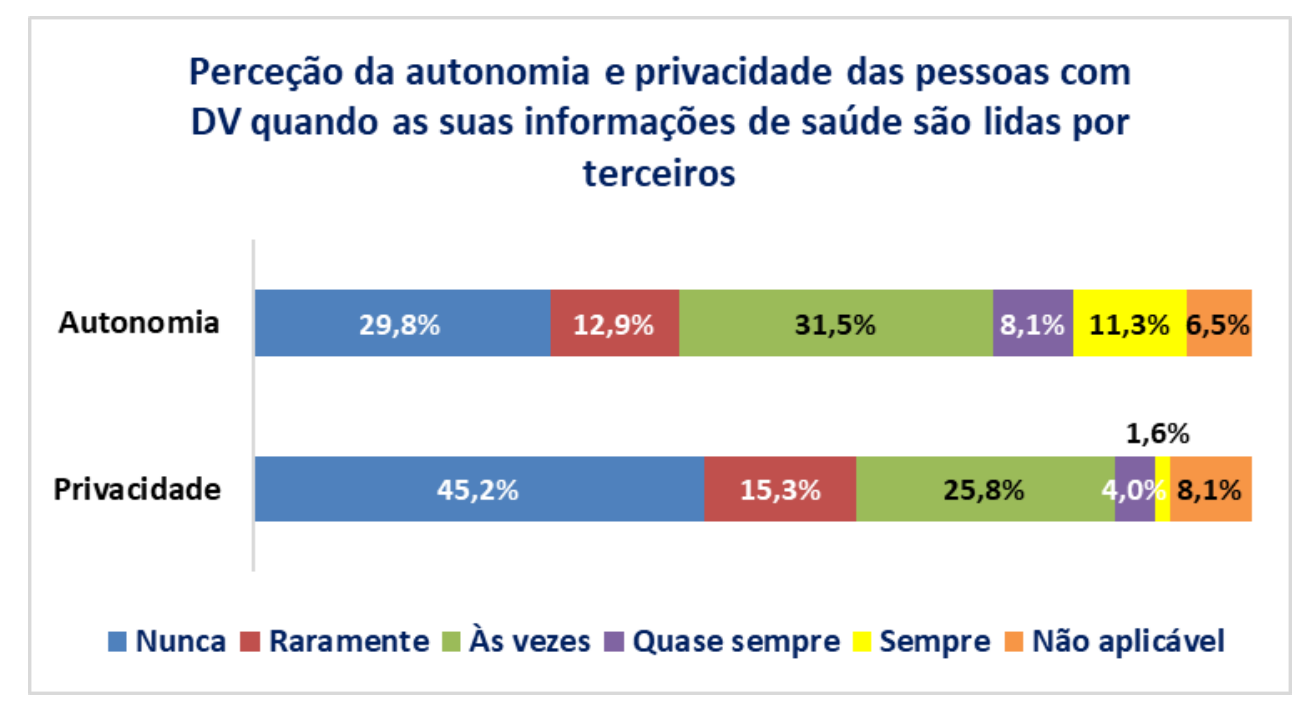

Fonte: Elaboração própria.

Gráfico 3 - Resultados referentes à perceção das pessoas com DV relativamente à sua autonomia e privacidade poder ser posta em causa pelo facto de outras pessoas lhes lerem informações relacionadas com a sua saúde. $\mathrm{N}=124$

\section{Interação com os profissionais}


Rev. Interd. em Cult. e Soc. (RICS), São Luís, v. 7, n. 1, p. 225 - 241, jan./jun. 2021 ISSN eletrônico: 2447-6498

No que se refere à interação com os profissionais, $65 \%$ dos participantes consideram que é "muito importante" os atos médicos serem descritos, previamente, pelos profissionais. Um grupo de $57 \%$ dos inquiridos diz que os especialistas o fazem "sempre" e $21 \%$ "quase sempre". Uma parcela de $15 \%$ dos participantes afirma que os profissionais o fazem “às vezes". De acordo com vários autores (e.g. CUPPLES et al, 2012, THURSTON, THURSTON, 2010), esta postura dos profissionais facilita o diálogo com os utentes com DV porque permite a compreensão do que irá suceder-se e contribui para a diminuição de receios relacionados com o desconhecido. Traz ainda segurança, tranquilidade e familiaridade. (O’DAY, KILLEEN, IEZZONI, 2004; IEZZONI, O’DAY, 2006; SIBLEY, 2009).

Quando as pessoas com DV estão num gabinete com mais de um profissional, a maioria dos participantes considera importante que esses profissionais sejam identificados. Esta atitude permite que a pessoa com DV se sinta mais confortável e confiante preservando a individualidade. (e.g. MEDEIROS et al, 2017; CUPPLES et al, 2012).

A maioria dos participantes considera relevante registrar notas relacionadas a consultas e tratamentos, para as consultarem posteriormente. Autores como O'Day, Killeen e Iezzoni (2004) e Cupples, Hart, Johnston e Jackson (2012) dão como exemplos a gravação de consultas e existir um tempo extra para que a pessoa com DV possa anotar informações que de outra forma seriam explanadas num suporte inacessível.

Conforme pode ser visto no gráfico 4, um grupo significativo de participantes (21\%) constata que os profissionais falam diretamente para o acompanhante e não para as pessoas com DV, o que pode inibir a iniciativa destes em expor as suas necessidades. (SIBLEY, 2009, THURSTON; THURSTON, 2010; MEDEIROS et al, 2017).

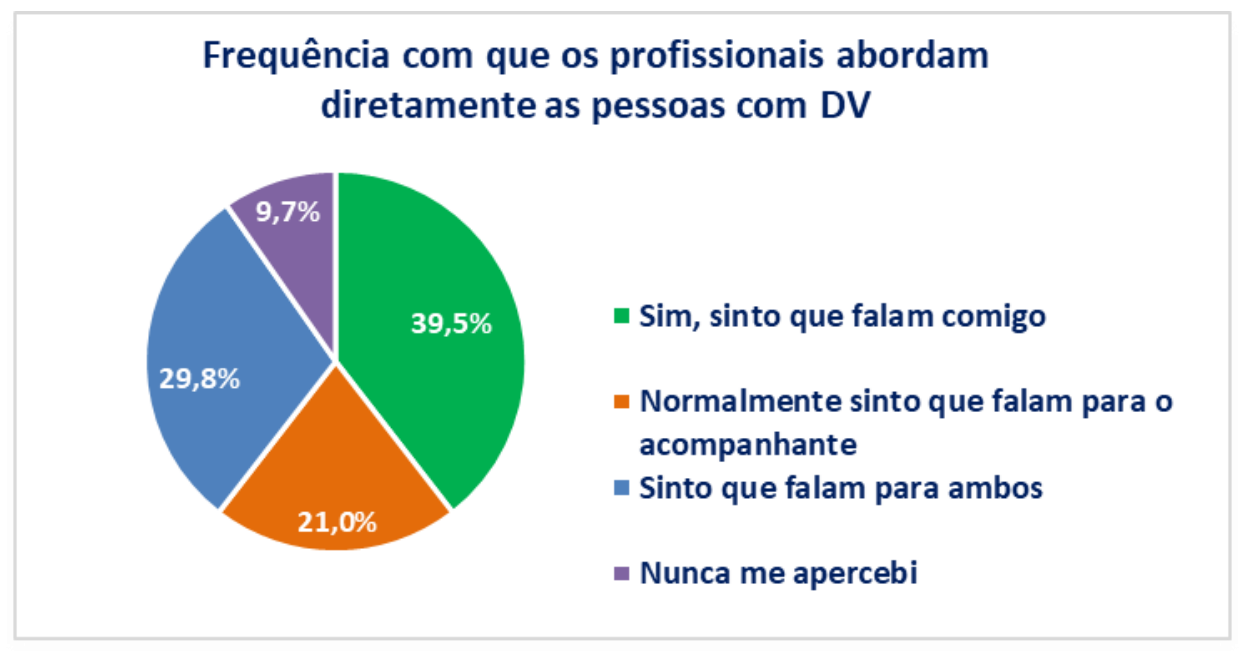

Fonte: Elaboração própria. 
Rev. Interd. em Cult. e Soc. (RICS), São Luís, v. 7, n. 1, p. 225 - 241, jan./jun. 2021

ISSN eletrônico: 2447-6498

Gráfico 4 - Resultados referentes à frequência com que os profissionais abordam diretamente as pessoas com DV, ainda que acompanhadas. $\mathrm{N}=124$

Quando apoiados por um profissional de saúde, a maioria dos participantes sente-se bem guiada, no entanto, quando têm de se deslocar de um ponto ao outro no CS - de forma autónoma - uma parte significativa dos inquiridos respondeu que nunca ou raramente recebem orientações claras que o permitam mover-se com segurança. Guiar uma pessoa com DV de modo digno e, ao mesmo tempo, usar linguagem clara, objetiva e esclarecedora facilita a comunicação, deixando-a mais segura e orientada. (IEZZONI; O’DAY, 2006; SIBLEY, 2009; THURSTON; THURSTON, 2010).

Uma percentagem significativa dos inquiridos afirma, nos casos em que necessitam de partilhar informações oralmente num espaço onde privam mais pessoas, não existir atenção dos serviços em assegurar medidas que acautelem a sua privacidade e promovam a confidencialidade dos seus dados pessoais.

\section{CONSIDERAÇÕES FINAIS}

Os resultados do estudo revelam ser importante que os serviços promovam a disponibilização de informação em formatos alternativos, por exemplo, em Braille, e-mail, SMS, texto ampliado, telefone, assim como o acesso a sites acessíveis. De modo geral, as pessoas com DV sentem necessidade de o atendimento ser mais flexível e personalizado, podendo deste modo ser equacionada, de forma organizada e atempada, a adoção de práticas e métodos que respondam às suas necessidades.

É, igualmente, importante encetar melhorias ao nível arquitetónico e metodológico que facilitem a orientação e mobilidade de todos os cidadãos, independente das características, nos edifícios, como por exemplo, da utilização de diferentes meios sonoros e/ou visuais (e.g. contraste cromático) no dispositivo de chamada dos utentes e também na disponibilização de apoio, por parte dos recursos humanos, para a deslocação e orientação no CS. A criação de um ambiente acolhedor, fácil de circular, ausente de obstáculos e com sinalética clara, facilita a orientação e mobilidade das pessoas com DV.

De forma a prestar melhores cuidados, é essencial apostar em formação e sensibilização de profissionais da saúde para o atendimento das pessoas com DV, no que se refere à 
Rev. Interd. em Cult. e Soc. (RICS), São Luís, v. 7, n. 1, p. 225 - 241, jan./jun. 2021 ISSN eletrônico: 2447-6498

forma como a DV afeta o seu dia-a-dia, como se expressa e qual o impacto no nível da comunicação e interação com os outros. É essencial estabelecer uma comunicação verbal clara e objetiva na interação face a face no decorrer do atendimento e acompanhamento (e.g. atos clínicos, administrativos, apoio à deslocação). Igualmente importante é compreender as diferentes necessidades das pessoas com DV no acesso à informação escrita e de que modo o desempenho autónomo, a privacidade e autogestão das informações relacionadas com a sua saúde, podem ficar comprometidos quando não lhes são garantidas condições de acessibilidade.

A par desta sensibilização dos profissionais, também é relevante consciencializar as pessoas com DV e dotá-las de mais informação/legislação que lhes permita ter um papel mais ativo na defesa dos seus direitos e na tomada de decisões.

Por outro lado, as organizações são fundamentais para o sucesso da comunicação dos seus serviços, na medida em devem promover uma cultura organizacional, conhecida por todos os colaboradores, que facilite a disponibilização de informação, procedimentos e recursos materiais que respondam à diversidade de necessidades das pessoas com DV. Portanto, que concretize apoios personalizados em termos de preferências de leitura, entre outros aspetos relevantes na comunicação. Esta dinâmica depreende que os suportes alternativos não sejam vistos como um pedido especial/diferente de uma pessoa em concreto, mas antes como mais uma oferta dos serviços, desmistificando eventuais preconceitos. Paralelamente, é imperativo que as organizações criem espaços físicos mais acessíveis e, sempre que possível, envolvendo as próprias pessoas com DV como testers nos seus planos de acessibilidade. É igualmente crucial as instituições considerarem a acessibilidade dos seus sites na $W E B$, recurso imprescindível nos dias de hoje para veicular informação acessível às pessoas com DV.

A capacitação dos serviços para a promoção de uma comunicação eficaz junto das pessoas com DV é importante para que estas possam aceder à informação de forma acessível e tomar decisões esclarecidas sobre a sua saúde, promovendo desta forma a autonomia no acesso aos cuidados de saúde primários.

\section{REFERÊNCIAS}


Rev. Interd. em Cult. e Soc. (RICS), São Luís, v. 7, n. 1, p. 225 - 241, jan./jun. 2021

ISSN eletrônico: 2447-6498

ALMEIDA, C. V.; SILVA, C. R.; ROSADO, D.; MIRANDA, D.; OLIVEIRA, D.; MATA, F.; ASSUNÇÃO, V. Manual de boas práticas. Literacia em saúde - Capacitação dos profissionais de saúde. Direção Geral da Saúde, 2019.

ALMEIDA, J.; PINTO, J. A investigação nas ciências sociais. Editora Presença, 1995. ASSOCIAÇÃO DOS CEGOS E AMBLÍOPES DE PORTUGAL. (s.d.). Recomendações técnicas ACAPO: Como criar serviços de atendimento mais acessíveis (volume 6). Recuperado em 04 de abril de http://www.acapo.pt/deficiencia-visual/documentos-epublicacoes/recomendacoes-tecnicas/como-criar-servicos-de.

ARRIAGA, M. T.; SANTOS, B.; SILVA, A.; MATA, F.; CHAVES, N.; FREITAS, G. Plano de ação para a literacia em saúde 2019-2021 - Portugal. Ministério da Saúde, Direção-Geral da Saúde, 2019.

CRESWELL, J. W. Projeto de pesquisa - Métodos qualitativo, quantitativo e misto. $2^{\circ}$ edição. Porto Alegre, ArteMed, 2007.

CUPLES, M.; HART, P.; JOHNSTON, A.; JACKSON, A. Improving healthcare acess for people with visual impairment and blindness. BMJ Clinical Research Edition, 2012, 344(7842), 1-5.

ESPANHA, R.; ÁVILA, P.; MENDES, R. Literacia em saúde em Portugal. Lisboa, Fundação Calouste Gulbenkian, 2016.

FORTIN, M. O processo de investigação: da concepção à realização. Louries, Lusociência, 2009.

IEZZONI, L.; O’DAY, B. More Than Ramps: A Guide to Improving Health Care Quality and Access for People with Disabilities. Oxford University Press, 2006.

INTERACTION DESIGN FOUNDATION (S.D.). Accessibility: What is Accessibility. Recuperado em 04 de abril de https://www.interactiondesign.org/literature/topics/accessibility.

MEDEIROS, T.; COSTA, K.; COSTA, T.; MARTINS, K.; DANTAS, T. Acessibilidade de pessoas com deficiência visual nos serviços de saúde. Revista enfermagem UERJ, 2017, 25(e11424), 1-5.

MINISTÉRIO PÚBLICO DE PORTUGAL. Convenção sobre os Direitos das Pessoas com Deficiência (texto em português). Procuradoria-Geral da República, Gabinete de Documentação e Direito Comparado, 2009. Retirado de

http://www.ministeriopublico.pt/instrumento/convencao-sobre-os-direitos-das-pessoas-comdeficiencia

NZEGWU, F. The experiencie of blind and partially sighted users of the NHS: Making the delivery of care more inclusive and effective. International Congress Series, 2005, 1282, 230-234. 
Rev. Interd. em Cult. e Soc. (RICS), São Luís, v. 7, n. 1, p. 225 - 241, jan./jun. 2021 ISSN eletrônico: 2447-6498

O'DAY, B.L.; KILLEEN, M.; IEZZONI, L.I. Improving health care experiences of persons who are blind or have low vision: suggestions from focus groups. American Journal of Medical Quality, 2004, 19, 193-200.

PAIVA, D.; SILVA, S.; SEVERO, M.; MOURA-FERREIRA, P.; LUNET, N.; AZEVEDO, A. Prevalência de Literacia em Saúde Inadequada em Portugal Medida com o Newest Vital Sign. Acta Médica Portuguesa, 30(12), 861-869, 2017.

PEREIRA, R.; FREIRE, C. A importância da informação acessível para uma melhor literacia de saúde. In C. Freire, C. Mangas \& J. Sousa (Org.) Livro de Resumos da VI Conferência Internacional para a Inclusão - 2020 (pp. 505-511). https://includit.ipleiria.pt/.

PEREIRA, R. O acesso aos cuidados de saúde primários - A perceção das pessoas com DV [Dissertação de mestrado não publicada]. Leiria, Instituto Politécnico de Leiria, 2020. Disponível a partir de 〈https://iconline.ipleiria.pt/handle/10400.8/5031〉.

REGULAMENTO (UE) 2016/ 679. Proteção das pessoas singulares no que diz respeito ao tratamento de dados pessoais e à livre circulação desses dados e que revoga a Diretiva 95/46/CE (Regulamento Geral sobre a Proteção de Dados). Jornal Oficial da União Europeia, 2016.

RESOLUÇÃO DA ASSEMBLEIA DA REPÚBLICA No56/2009. Convenção sobre os direitos das pessoas com Deficiência. Diário da República 1. a série. N. ${ }^{\circ} 146,2009$ (09-0730), 4906-4929.

SASSAKI, R. K. Inclusão: acessibilidade no lazer, trabalho e educação. Revista Nacional de Reabilitação - Reação, 2009, (pp.10-16).

SIBLEY, E. Towards an inclusive health service: a report into the availability of health information for blind and partially sighted people. Centre for Educational and Psychosocial Research for RNIB Scotland, 2009.

THURSTON, M.; THURSTON, A. Accessibility of health information for blind and partially sighted people. Centre for Educational and Psychosocial Research for RNIB Scotland, 2010. 